OPEN ACCESS

Edited by:

Subhash C. Verma,

University of Nevada, Reno,

United States

Reviewed by:

Neelam Sharma-Walia,

Rosalind Franklin University of

Medicine and Science, United States

Qiliang Cai,

Fudan University, China

*Correspondence:

Zhiqiang Qin

zqin@uams.edu

Luis Del Valle

Idelva@Isuhsc.edu

Specialty section:

This article was submitted to Virus and Host,

a section of the journal

Frontiers in Cellular

and Infection Microbiology

Received: 27 July 2020 Accepted: 13 October 2020 Published: 30 October 2020

Citation:

Barrett L, Chen J, Dai L, Plaisance-Bonstaff $K$, Del Valle $L$ and Qin Z (2020) Role of Interleukin-1

Family Members and Signaling Pathways in KSHV Pathogenesis. Front. Cell. Infect. Microbiol. 10:587929. doi: 10.3389/fcimb.2020.587929

\section{Role of Interleukin-1 Family Members and Signaling Pathways in KSHV Pathogenesis}

\author{
Lindsey Barrett ${ }^{1}$, Jungang Chen ${ }^{1}$, Lu Dai ${ }^{1}$, Karlie Plaisance-Bonstaff ${ }^{2}$, Luis Del Valle ${ }^{3 *}$ \\ and Zhiqiang Qin $^{1 *}$ \\ ${ }^{1}$ Department of Pathology, Winthrop P. Rockefeller Cancer Institute, University of Arkansas for Medical Sciences, Little Rock, \\ AR, United States, ${ }^{2}$ Department of Medicine, Louisiana State University Health Sciences Center, Louisiana Cancer Research \\ Center, New Orleans, LA, United States, ${ }^{3}$ Department of Pathology, Louisiana State University Health Sciences Center, \\ Louisiana Cancer Research Center, New Orleans, LA, United States
}

Kaposi's sarcoma-associated herpesvirus (KSHV) represents the etiological agent for several human malignancies, including Kaposi's sarcoma (KS), primary effusion lymphoma (PEL), and multicentric Castleman's disease (MCD), which are mostly seen in immunocompromised patients. In fact, KSHV has developed many strategies to hijack host immune response, including the regulation of inflammatory cytokine production. Interleukin-1 (IL-1) family represents a major mediator for inflammation and plays an important role in both innate and adaptive immunity. Furthermore, a broadening list of diseases has revealed the pathologic role of IL-1 mediated inflammation. In the current mini-review, we have summarized recent findings about how this oncogenic virus is able to manipulate the activities of IL-1 signaling pathway to facilitate disease progression. We also discuss the therapeutic potential of IL-1 blockade against KSHV-related diseases and several unsolved questions in this interesting field.

Keywords: Kaposi's sarcoma-associated herpesvirus, Kaposi's sarcoma, primary effusion lymphoma, multicentric Castleman's disease, interleukin-1

\section{INTRODUCTION}

Kaposi's sarcoma-associated herpesvirus (KSHV) infection causes several human cancers including Kaposi's sarcoma (KS), primary effusion lymphoma (PEL), and multicentric Castleman's disease (MCD) (Broussard and Damania, 2019). These KSHV-associated malignancies develop mainly in immunocompromised patients, especially those infected with human immunodeficiency viruses (HIVs) (Vangipuram and Tyring, 2019). Further, the morbidity rate of patients with KSHVassociated diseases is much higher in patients with compromised immune systems compared to those with competent immune systems (Mesri et al., 2010).

KSHV has two alternating life-cycle programs following primary infection of host cells, the latent and lytic phases, which are characterized by different patterns of viral gene expression (Mesri et al., 2010). During latency, viral genomes persist as circular episomes with no progeny virion production and only a limited number of latency-associated genes expressed, including latency-associated nuclear antigen (LANA), viral Fas-associated protein with death domain (FADD)-like interleukin$1 \beta$-converting enzyme (FLICE)-like inhibitory protein (vFLIP), viral cyclin (vCyclin), as well as 
some viral microRNAs (Uppal et al., 2014). Once entering the lytic phase, which is caused by various stimuli, almost all viral genes are highly expressed, followed by genomic DNA replication and mature virion release (Ye et al., 2011). KSHV is known to hijack many aspects of the host's immune response such as viral detection and cytokine production. Interleukin-1 (IL-1) is an inflammatory cytokine family of 11 distinct proteins that has a wide array of functions in innate immunity processes. The IL-1 superfamily contains many pro-inflammatory cytokines (IL-1 $\alpha$, IL-1 $\beta$, IL-18, IL-33, IL-36 $\alpha$, IL-36 $\beta$, and IL$36 \gamma$ ) and a few anti-inflammatory cytokines (IL-36Ra, IL-37, and IL-38) (Boraschi and Tagliabue, 2013). Among them, IL-1 is the defining member of this family and its physiology and relationship to pathology has been thoroughly studied and reported. IL-1 includes two activator cytokines, IL- $1 \alpha$ and IL$1 \beta$, and one inhibitory factor, the IL- 1 receptor antagonist (named as IL-1Ra). The main function of IL-1 is to respond to tissue damage caused by pathogen-associated molecular patterns (PAMPs) such as viral products, or damage-associated molecular patterns (DAMPs) such as adenosine 5'-triphosphate (Xu et al., 2019). Upon stimulation, IL- $1 \alpha$ and IL- $1 \beta$ both bind to the type I IL-1 receptor (IL-1R1) which then recruits the IL-1 receptor accessory protein (IL-1RAP), as well as the adaptor protein MyD88, which are necessary for triggering signal transduction. Once the IL-1 receptor complex is formed, a downstream signaling cascade is activated which then stimulates a collection of related immune responses and/or inflammatory genes (Jensen, 2017). Dysregulation of the IL-1 pathway has been shown to be linked to a number of autoinflammatory and autoimmune diseases, such as atherosclerosis and systemic sclerosis, respectively, as well as cancers like gastric carcinoma and lung cancer (El-Omar et al., 2001; Bhat et al., 2014; Ridker et al., 2017; Xu et al., 2019).

KSHV infection has been found to induce the production of a variety of host pro-inflammatory cytokines. For example, primary KSHV infection in monocytes can increase the release of IL-1 $\alpha$, IL-1, and IL-6 (Host et al., 2017). These cytokines have been suggested to regulate early KS lesion progression and have been found at high levels in the sera of KS patients (Ensoli and Stürzl, 1998). Other IL-1 family members such as IL-33 have recently been shown to play a role in $\mathrm{KSHV}$ pathogenesis by regulating chromatin compaction through nucleosome-nucleosome interactions (Roussel et al., 2008). Therefore, in this mini-review, we will summarize recent findings about the relationship between KSHV and the IL-1 family members. We will try to highlight how KSHV may utilize the IL-1 signaling pathway to facilitate disease progression and how potential immunotherapies could target such mechanisms.

\section{THE IL-1 SIGNALING PATHWAY}

IL-1 is a major mediator for inflammation and plays an important role in both innate and adaptive immunity. IL- $1 \alpha$ and IL-1 $\beta$ both signal through the cell surface receptor, IL-1R1
(Jensen, 2017). Upon ligand binding, the transmembrane IL-1R accessory protein, IL-1RAP, is recruited to the site. This heterodimer formation leads to intracellular recruitment of the adaptor protein, MyD88, and mobilization of IL-1R-associated kinases (e.g., IRAK1, IRAK2, and IRAK4). These kinases, along with additional signaling factors, lead to the phosphorylation and degradation of nuclear factor $\kappa \mathrm{B}(\mathrm{NF}-\kappa \mathrm{B})$ inhibitor I $\mathrm{KB}$. The end of this signaling pathway results in the translocation of activated transcription factors, such as activator protein 1 (AP-1) and NF$\mathrm{KB}$, to the nucleus where specific gene expression is activated. IL18 and IL-33 also stimulate gene expression through the same intracellular pathway using their receptor-accessory protein complexes (IL-18R1/IL-18RAP and IL-1R-like 1/IL-1RAP). The other cytokines, IL-36 $\alpha$, IL-36 $\beta$, and IL-36 $\gamma$, bind to the receptor IL-1R-like 2 (IL-1RL2 or IL-36R), which then uses IL1RAP as its receptor-accessory protein and stimulates the same signaling cascade as IL-1, IL-18, and IL-33 (Dinarello, 2019). Interestingly, KSHV has developed strategies to manipulate the functions of b1these different IL-1 signaling molecules after invading host cells.

\section{IL-1 $\alpha / \beta$ AND RECEPTORS}

Several studies have reported that KSHV infection or viral protein infiltration can upregulate IL- $1 \alpha$ and/or IL- $1 \beta$ expression. For instance, one study showed that ectopic expression of viral macrophage inflammatory protein-II (vMIP-II) within endothelial cells upregulated multiple proangiogenic factors, including IL- $1 \alpha$, resulting in enhanced angiogenesis (Cherqui et al., 2007). Another viral protein, vOX2, a glycosylated cell surface protein, was found to dramatically stimulate primary monocytes, macrophages, and dendritic cells to produce IL-1 $\beta$ (Chung et al., 2002). On the other hand, KSHV Orf63, encoding a viral homolog of human NLRP1 (NACHT, LRR, FIIND, CARD domain and PYD domains-containing protein 1), was found to reduce IL- $1 \beta$ expression and related signaling through inhibition of the inflammasome (Gregory et al., 2011). In fact, many studies suggest that inflammatory and angiogenic cytokines including IL-1 $\beta$ contribute to the pathogenesis of KS by causing abnormal proliferation, angiogenesis, and a KS-like phenotype independent of KSHV (Ensoli et al., 1992). For example, IL-1 $\beta$ was markedly elevated in most KS lesions (Samaniego et al., 1997). Furthermore, IL-1 $\beta$ was elevated during initial KSHV-MCD flares compared with remission (Polizzotto et al., 2013). Interestingly, our recent study demonstrated that IL- $1 \beta$ was required for the upregulation of PD-L1 expression by viral lytic reactivation from KSHV-infected tumor cells (Chen et al., 2019), which may represent a novel mechanism for virus-associated tumor cell immune escape. It remains unclear about the situation of receptor and accessory proteins of IL-1 in KSHV-infected cells. Our recent data indicate that KSHV infection significantly upregulates IL1R1 and IL1RAP from endothelial cells (Figure 1A). Moreover, both proteins are found highly expressed in KS tumor cells, especially IL1R1 (Figure 1B). 

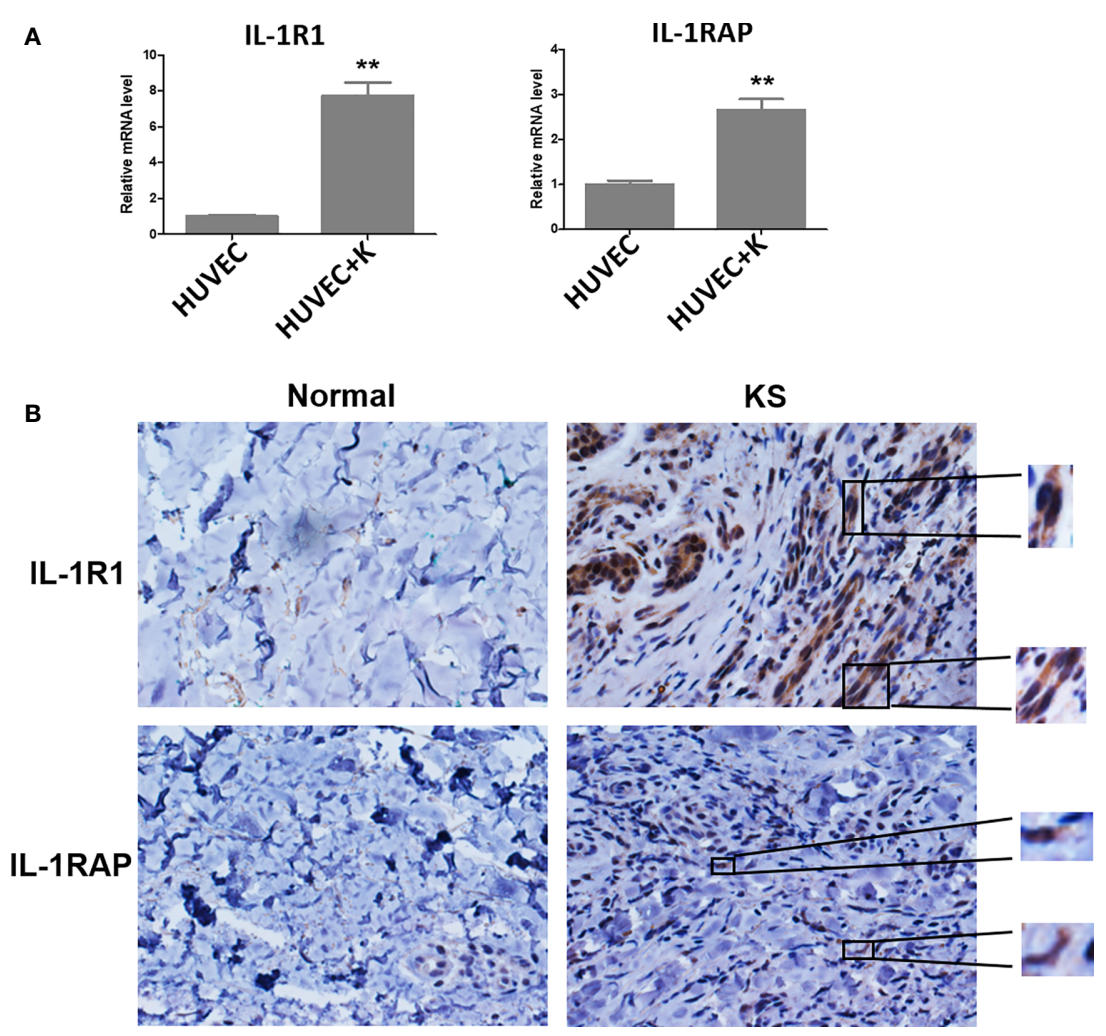

FIGURE 1 | IL-1R1 and IL-1RAP are upregulated by KSHV and highly expressed within AIDS-KS tumor tissues. (A) Primary human umbilical vein endothelial cells (HUVEC) were infected with KSHV (MOI 10) or not for $48 \mathrm{~h}$, followed by qRT-PCR analysis. The data were normalized to the $\beta$-actin housekeeping gene expression. Error bars represent the S.D. for three independent experiments. ${ }^{* *} \mathrm{P}<0.01$. (B) Expression of IL-1R1 and IL-1RAP in representative formalin-fixed paraffinembedded KS tissues from HIV+ patient without treatment were determined by immunohistochemical staining (40x). The higher magnification for IL-1R1 and IL1RAP detection in KS tumor cells were also shown (60x). Normal skin tissues were used as a control.

\section{IL-18, IL-33, AND IL-36}

KSHV latency, especially viral FADD-like interleukin-1- $\beta-$ converting enzyme [FLICE/caspase 8]-inhibitory protein (vFLIP), was found to induce the expression of IL-18 (as well as IL-1 $\beta$ ) in an NF- $\mathrm{KB}$ dependent manner (Singh et al., 2013). In contrast, one of the viral lytic products, KSHV polyadenylated nuclear RNA (PAN RNA) decreased the expression of IL-18 (Rossetto and Pari, 2011). Similar inhibitory effects on IL-18 were observed with KSHV Orf63 in the study mentioned above (Gregory et al., 2011).

The functional roles of IL-33 and IL-36, and their regulatory mechanisms in KSHV-infected cells remain mostly unclear. One very recent study reported that the plasma IL-33 concentrations were higher in individuals with KS in Uganda, Africa (Byakwaga et al., 2020), implying that this cytokine and its related signaling may also play role in KSHV pathogenesis. Interestingly, IL-33 has also been demonstrated as a chromatin-associated factor in the nucleus of endothelial cells, which has a short chromatinbinding peptide that shares similarities with a motif found in KSHV-encoded latency-associated nuclear antigen (LANA) (Carriere et al., 2007; Roussel et al., 2008). As we know, LANA is responsible for the attachment of the viral episome to mitotic chromosomes (Barbera et al., 2006); thus, this IL-33 peptide can also dock into the acidic pocket formed by the H2A-H2B dimer at the nucleosomal surface and regulate chromatin compaction through nucleosome-nucleosome interactions. However, the association between IL-33 and KSHV latency and lytic reactivation remains unknown.

\section{MYD88 AND IRAKs}

One study using X chromosome-targeted sequencing identified 34 common missense mutations in 100\% of PEL cases, including a Phe196Ser change in the IRAK1 protein. Moreover, IRAK1 was constitutively phosphorylated in PEL and required for tumor cell survival (Yang et al., 2014). By using CRISPR/Cas9 knockout technology, the same group recently reported that established PEL cell lines were able to circumvent the loss of IRAK1, IRAK4, and MyD88, while the deletion clones were deficient in IL-10 production (Seltzer et al., 2020). Due to the suppression of T cell function by IL-10, the authors suggest that the IRAK pathway may contribute to early-stage development of PEL. KSHV 
encodes 12 pre-microRNAs (pre-miRNAs), which are processed into 25 mature microRNAs (miRNAs) (Qin et al., 2017). Interestingly, Abend et al. reported that IRAK1 and MyD88 were directly targeted by several KSHV-microRNAs, particularly miR-K12-9 and miR-K12-5, respectively (Abend et al., 2012). The presence of miR-K12-9 and miR-K12-5 inhibited the production of IL- 6 and IL- 8 upon IL- $1 \alpha$ stimulation of endothelial cells. In another study, Lingel et al. reported that KSHV-encoded replication and transcription activator (RTA) was able to bind to MyD88 RNA and suppress its RNA synthesis (Lingel et al., 2016). Another group recently found that KSHV RTA downregulated MyD88 expression at the protein level by degrading MyD88 through the ubiquitin (Ub)-proteasome pathway (Zhao et al., 2015).

\section{IL-1 BLOCKADE}

Since IL-1 is a master cytokine of local and systemic inflammation, pharmacological blockade of IL-1 activity has been applied in a variety of inflammatory diseases that results in a rapid and sustained reduction in disease severity. There are three major categories of IL-1 blockers which have been approved by the Food and Drug Administration (FDA) for clinical treatment: 1) The IL-1 receptor antagonist (e.g., Anakinra), blocks the IL-1 receptor and therefore reduces the activity of IL- $1 \alpha$ and IL-1 $\beta ; 2$ ) The soluble decoy receptor (e.g., Rilonacept, also known as IL-1 Trap), a dimeric fusion protein consisting of the ligand-binding domains of the extracellular portions of IL-1R1 and IL-1RAP linked in-line to the fragmentcrystallizable portion (Fc region) of human IgG1 that binds and neutralizes IL-1; 3) The neutralizing monoclonal anti-IL-1 $\beta$ antibody (e.g., Canakinumab), which directly targets IL-1 $\beta$ (Dinarello and van der Meer, 2013; Dinarello, 2013). There are other neutralizing monoclonal antibodies targeting IL- $1 \alpha$ or the IL-1 receptor in different clinical trials (Dinarello and van der Meer, 2013). Interestingly, Boehringer Ingelheim Company recently developed a new IL1RAP antibody, BI-5041, which targets a unique epitope on IL-1RAP and therefore blocks IL-1, IL-33, and IL-36 signalling. Currently, there is limited data about IL-1 blockade therapy in KSHV-related malignancies (El-Osta et al., 2010). Two case reports detailed the successful treatment of MCD by Anakinra for two patients, although their KSHV infection status remains unclear (Galeotti et al., 2008; El-Osta et al., 2010).

\section{CONCLUSION AND PROSPECTIVE}

Current research reveals that KSHV has developed different strategies to manipulate IL-1 signaling activity (summarized in Figure 2) in order to balance the host's inflammatory response or help the virus escape the host's immune response. The virusencoded latent and lytic proteins and even viral non-coding RNAs can target multiple components of the IL-1 signaling pathway. However, there are still many questions in this field waiting for further investigation. For example, the functions and regulatory mechanisms of certain IL-1 family members (e.g., IL-36, IL-37, IL$1 \mathrm{Ra}$, and IL-1RAP) during KSHV infection or virus-induced tumorigenesis remain unknown. It is also unclear whether the intermediates of IL-1 signaling may affect KSHV replication, especially the "latency to lytic" switch. Furthermore, the efficacy

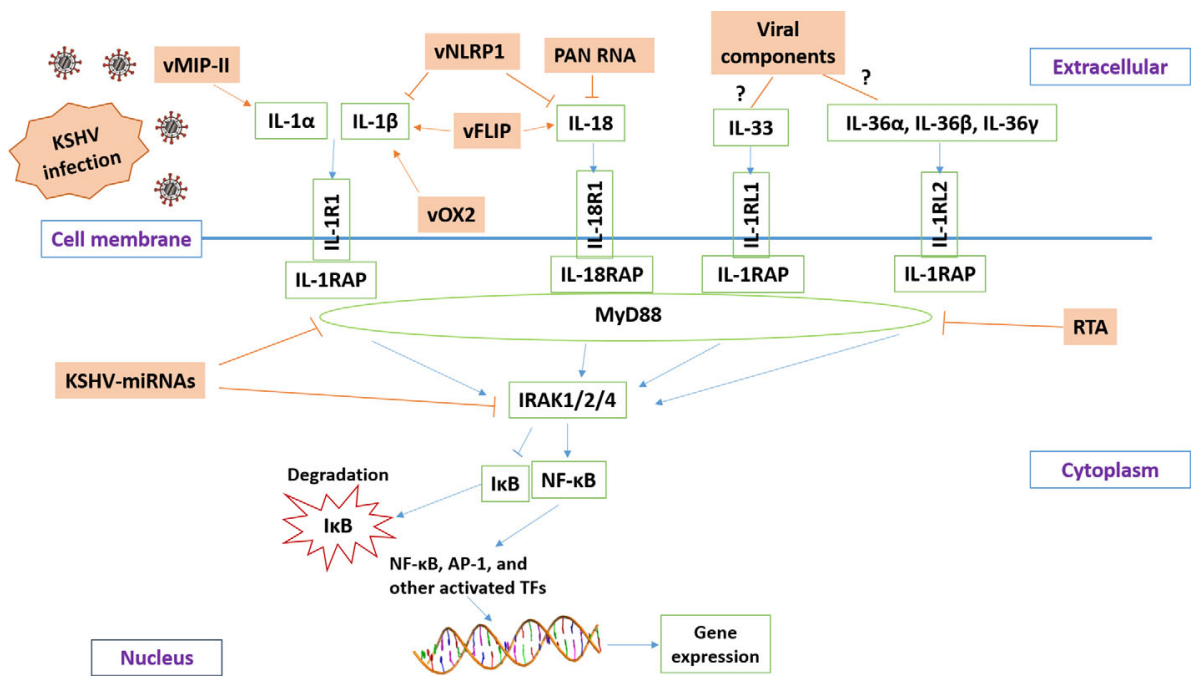

FIGURE 2 | Schematic diagram of potential mechanisms for KSHV manipulating IL-1 signaling pathway. The brown and green rectangles represent viral and host genes, respectively. The arrows and bars represent the activation and inhibition, respectively. Notably, the mechanisms of KSHV regulation of IL-33 and IL-36 signaling remain mostly unknown. 
of IL-1 blockade therapy either alone or combined with other therapies for KSHV-related malignancies needs to be tested. Fully understanding these questions will shed light on the molecular mechanisms of KSHV pathogenesis and tumorigenesis and facilitate the development of more efficacious antiviral and anticancer treatments.

\section{AUTHOR CONTRIBUTIONS}

LB wrote the manuscript. JC, LD, and KP-B participated in figure preparation and discussion. LV and ZQ reviewed and edited the

\section{REFERENCES}

Abend, J. R., Ramalingam, D., Kieffer-Kwon, P., Uldrick, T. S., Yarchoan, R., and Ziegelbauer, J. M. (2012). Kaposi's sarcoma-associated herpesvirus microRNAs target IRAK1 and MYD88, two components of the toll-like receptor/ interleukin-1R signaling cascade, to reduce inflammatory-cytokine expression. J. Virol. 86, 11663-11674. doi: 10.1128/JVI.01147-12

Barbera, A. J., Chodaparambil, J. V., Kelley-Clarke, B., Joukov, V., Walter, J. C., Luger, K., et al. (2006). The nucleosomal surface as a docking station for Kaposi's sarcoma herpesvirus LANA. Science 311, 856-861. doi: 10.1126/ science.1120541

Bhat, I. A., Naykoo, N. A., Qasim, I., Ganie, F. A., Yousuf, Q., Bhat, B. A., et al. (2014). Association of interleukin 1 beta (IL-1 $\beta$ ) polymorphism with mRNA expression and risk of non-small lung cancer. Meta Gene 2, 123-133. doi: 10.1016/j.mgene.2013.12.002

Boraschi, D., and Tagliabue, A. (2013). The interleukin-1 receptor family. Semin. Immunol. 25, 394-407. doi: 10.1016/j.smim.2013.10.023

Broussard, G., and Damania, B. (2019). KSHV: Immune Modulation and Immunotherapy. Front. Immunol. 10:3084. doi: 10.3389/fimmu.2019.03084

Byakwaga, H., Barbachano-Guerrero, A., Wang, D., Mcallister, S., Naphri, K., Laker-Oketta, M., et al. (2020). Immunoglobulin E (IgE) Levels are associated with Kaposi's Sarcoma in HIV-Infected African Adults. J. Infect. Dis. doi: 10.1093/infdis/jiaa340

Carriere, V., Roussel, L., Ortega, N., Lacorre, D. A., Americh, L., Aguilar, L., et al. (2007). IL-33, the IL-1-like cytokine ligand for ST2 receptor, is a chromatinassociated nuclear factor in vivo. Proc. Natl. Acad. Sci. U. S. A. 104, 282-287. doi: 10.1073/pnas.0606854104

Chen, J., Del Valle, L., Lin, H. Y., Plaisance-Bonstaff, K., Forrest, J. C., Post, S. R., et al. (2019). Expression of PD-1 and PD-Ls in Kaposi's sarcoma and regulation by oncogenic herpesvirus lytic reactivation. Virology 536, 16-19. doi: 10.1016/j.virol.2019.07.024

Cherqui, S., Kingdon, K. M., Thorpe, C., Kurian, S. M., and Salomon, D. R. (2007). Lentiviral gene delivery of vMIP-II to transplanted endothelial cells and endothelial progenitors is proangiogenic in vivo. Mol. Ther. 15, 1264-1272. doi: 10.1038/sj.mt.6300183

Chung, Y. H., Means, R. E., Choi, J. K., Lee, B. S., and Jung, J. U. (2002). Kaposi's sarcoma-associated herpesvirus OX2 glycoprotein activates myeloid-lineage cells to induce inflammatory cytokine production. J. Virol. 76, 4688-4698. doi: 10.1128/JVI.76.10.4688-4698.2002

Dinarello, C. A., and van der Meer, J. W. (2013). Treating inflammation by blocking interleukin-1 in humans. Semin. Immunol. 25, 469-484. doi: 10.1016/ j.smim.2013.10.008

Dinarello, C. A. (2013). Overview of the interleukin-1 family of ligands and receptors. Semin. Immunol. 25, 389-393. doi: 10.1016/j.smim.2013. 10.001

Dinarello, C. A. (2019). The IL-1 family of cytokines and receptors in rheumatic diseases. Nat. Rev. Rheumatol. 15, 612-632. doi: 10.1038/s41584-019-0277-8

El-Omar, E. M., Carrington, M., Chow, W. H., Mccoll, K. E., Bream, J. H., Young, H. A., et al. (2001). The role of interleukin-1 polymorphisms in the pathogenesis of gastric cancer. Nature 412, 99. doi: 10.1038/35083631 manuscript. All authors contributed to the article and approved the submitted version.

\section{FUNDING}

This work was supported by the Arkansas Bioscience Institute, the major research component of the Arkansas Tobacco Settlement Proceeds Act of 2000, and Winthrop P. Rockefeller Cancer Institute Core Facility Voucher Award. Funding sources had no role in study design, data collection and analysis, decision to publish, or preparation of the manuscript.

El-Osta, H., Janku, F., and Kurzrock, R. (2010). Successful treatment of Castleman's disease with interleukin-1 receptor antagonist (Anakinra). Mol. Cancer Ther. 9, 1485-1488. doi: 10.1158/1535-7163.MCT-10-0156

Ensoli, B., and Stürzl, M. (1998). Kaposi's sarcoma: a result of the interplay among inflammatory cytokines, angiogenic factors and viral agents. Cytokine Growth Factor Rev. 1, 63-83. doi: 10.1016/S1359-6101(97)00037-3

Ensoli, B., Barillari, G., and Gallo, R. C. (1992). Cytokines and growth factors in the pathogenesis of AIDS-associated Kaposi's sarcoma. Immunol. Rev. 127, 147-155. doi: 10.1111/j.1600-065X.1992.tb01412.x

Galeotti, C., Tran, T. A., Franchi-Abella, S., Fabre, M., Pariente, D., and KonePaut, I. (2008). IL-1RA agonist (anakinra) in the treatment of multifocal castleman disease: case report. J. Pediatr. Hematol. Oncol. 30, 920-924. doi: 10.1097/MPH.0b013e31818ab31f

Gregory, S. M., Davis, B. K., West, J. A., Taxman, D. J., Matsuzawa, S., Reed, J. C., et al. (2011). Discovery of a viral NLR homolog that inhibits the inflammasome. Science 331, 330-334. doi: 10.1126/science.1199478

Host, K. M., Jacobs, S. R., West, J. A., Zhang, Z., Costantini, L. M., Stopford, C. M., et al. (2017). Kaposi's Sarcoma-Associated Herpesvirus Increases PD-L1 and Proinflammatory Cytokine Expression in Human Monocytes. MBio 8, 17-29. doi: 10.1128/mBio.00917-17

Jensen, L. E. (2017). Interleukin-36 cytokines may overcome microbial immune evasion strategies that inhibit interleukin-1 family signaling. Sci. Signal 10, 3589-3600. doi: 10.1126/scisignal.aan3589

Lingel, A., Ehlers, E., Wang, Q., Cao, M., Wood, C., Lin, R., et al. (2016). Kaposi's Sarcoma-Associated Herpesvirus Reduces Cellular Myeloid Differentiation Primary-Response Gene 88 (MyD88) Expression via Modulation of Its RNA. J. Virol. 90, 180-188. doi: 10.1128/JVI.02342-15

Mesri, E. A., Cesarman, E., and Boshoff, C. (2010). Kaposi's sarcoma and its associated herpesvirus. Nat. Rev. Cancer 10, 707-719. doi: 10.1038/nrc2888

Polizzotto, M. N., Uldrick, T. S., Wang, V., Aleman, K., Wyvill, K. M., Marshall, V., et al. (2013). Human and viral interleukin-6 and other cytokines in Kaposi sarcoma herpesvirus-associated multicentric Castleman disease. Blood 122, 4189-4198. doi: 10.1182/blood-2013-08-519959

Qin, J., Li, W., Gao, S. J., and Lu, C. (2017). KSHV microRNAs: Tricks of the Devil. Trends Microbiol. 25, 648-661. doi: 10.1016/j.tim.2017.02.002

Ridker, P. M., Everett, B. M., Thuren, T., MacFadyen, J. G., Chang, W. H., Ballantyne, C., et al. (2017). Antiinflammatory Therapy with Canakinumab for Atherosclerotic disease. N. Engl. J. Med. 377, 1119-1131. doi: 10.1056/ NEJMoa1707914

Rossetto, C. C., and Pari, G. S. (2011). Kaposi's sarcoma-associated herpesvirus noncoding polyadenylated nuclear RNA interacts with virus- and host cellencoded proteins and suppresses expression of genes involved in immune modulation. J. Virol. 85, 13290-13297. doi: 10.1128/JVI.05886-11

Roussel, L., Erard, M., Cayrol, C., and Girard, J. P. (2008). Molecular mimicry between IL-33 and KSHV for attachment to chromatin through the H2A-H2B acidic pocket. EMBO Rep. 9, 1006-1012. doi: 10.1038/embor.2008.145

Samaniego, F., Markham, P. D., Gendelman, R., Gallo, R. C., and Ensoli, B. (1997). Inflammatory cytokines induce endothelial cells to produce and release basic fibroblast growth factor and to promote Kaposi's sarcoma-like lesions in nude mice. J. Immunol. 158, 1887-1894. 
Seltzer, J., Moorad, R., Schifano, J. M., Landis, J. T., and Dittmer, D. P. (2020). Interleukin-1 Receptor-Associated Kinase (IRAK) Signaling in Kaposi SarcomaAssociated Herpesvirus-Induced Primary Effusion Lymphoma. J. Virol. 94, 21232135. doi: 10.1128/JVI.02123-19

Singh, V. V., Kerur, N., Bottero, V., Dutta, S., Chakraborty, S., Ansari, M. A., et al. (2013). Kaposi's sarcoma-associated herpesvirus latency in endothelial and B cells activates gamma interferon-inducible protein 16-mediated inflammasomes. J. Virol. 87, 4417-4431. doi: 10.1128/JVI.03282-12

Uppal, T., Banerjee, S., Sun, Z., Verma, S. C., and Robertson, E. S. (2014). KSHV LANA-the master regulator of KSHV latency. Viruses 6, 4961-4998. doi: $10.3390 / \mathrm{v} 6124961$

Vangipuram, R., and Tyring, S. K. (2019). AIDS-Associated Malignancies. Cancer Treat Res. 177, 1-21. doi: 10.1007/978-3-030-03502-0_1

$\mathrm{Xu}, \mathrm{D} ., \mathrm{Mu}, \mathrm{R}$. , and Wei, X. (2019). The Roles of IL-1 Family Cytokines in the Pathogenesis of Systemic Sclerosis. Front. Immunol. 10, 2025. doi: 10.3389/ fimmu.2019.02025

Yang, D., Chen, W., Xiong, J., Sherrod, C. J., Henry, D. H., and Dittmer, D. P. (2014). Interleukin 1 receptor-associated kinase 1 (IRAK1) mutation is a common, essential driver for Kaposi sarcoma herpesvirus lymphoma. Proc. Natl. Acad. Sci. U. S. A. 111, E4762-E4768. doi: 10.1073/pnas.1405423111
Ye, F., Lei, X., and Gao, S. J. (2011). Mechanisms of Kaposi's Sarcoma-Associated Herpesvirus Latency and Reactivation. Adv. Virol. 2011, 1-19. doi: 10.1155/ 2011/193860

Zhao, Q., Liang, D., Sun, R., Jia, B., Xia, T., Xiao, H., et al. (2015). Kaposi's sarcoma-associated herpesvirus-encoded replication and transcription activator impairs innate immunity via ubiquitin-mediated degradation of myeloid differentiation factor 88. J. Virol. 89, 415-427. doi: 10.1128/ JVI.02591-14

Conflict of Interest: The authors declare that the research was conducted in the absence of any commercial or financial relationships that could be construed as a potential conflict of interest.

Copyright (C) 2020 Barrett, Chen, Dai, Plaisance-Bonstaff, Del Valle and Qin. This is an open-access article distributed under the terms of the Creative Commons Attribution License (CC BY). The use, distribution or reproduction in other forums is permitted, provided the original author(s) and the copyright owner(s) are credited and that the original publication in this journal is cited, in accordance with accepted academic practice. No use, distribution or reproduction is permitted which does not comply with these terms. 